\title{
Cadenas de valor globales en servicios: el caso de la industria de TI en México*
}

\author{
Global Value Chains in the Service Sector: the Mexican IT Industry Case
}

Flor Brown Grossman** y Lilia Domínguez Villalobos***

\section{RESUMEN}

El surgimiento de las cadenas de valor globales es resultado de un desplazamiento de la integración vertical las transnacionales a favor de las redes de proveedores externos. Lejos de la noción que identifica la subcontratación de servicios extraterritoriales con la manufactura, la evidencia muestra que aquélla ocupa $80 \%$ del total global, dentro del cual están los de tecnologías de la información (TI). Mediante estudios de caso se identifica el proceso mediante el cual las empresas de software se han podido insertar en la cadena de las Ti y los obstáculos a los que enfrentan una vez que ya están inmersas en ella, con la conjetura de que la oportunidad internacional se combinó con la utilización de apoyos de política, como el Prosoft, y la presencia de espíritu empresarial de sus dirigentes.

Palabras clave: cadenas de valor globales, globalización, industria de las tecnologías de la información, software, negocios internacionales.

Clasificación JEL: L86, F61, F68 y F23.

\begin{abstract}
The emergence of global value chains has come as a result of a shift away from vertically integrated transnational firms toward global outsourcing and the use of external supplier networks. Far from the notion that outsourcing of offshore services comes from manufacturing, evidence shows that services account for $80 \%$ of total offshore services on a global scale. Based on case study analysis this paper is an effort to identify the process by which firms are venturing into this Global Value Chain and the obstacles faced by firms, with the conjecture that the international opportunity combines with ad hoc programs for this industry and the presence of entrepreneurial spirit.
\end{abstract}

Keywords: global value chains, globalization, information technology industry, software, international business.

JEL classification: L86, F61, F68 y F23.

\footnotetext{
* Fecha de recepción: 07/10/2014. Fecha de aprobación: 08/05/2015. Este trabajo inicialmente fue parte del proyecto de investigación "Fragmentación de la producción e inserción de América Latina y el Caribe en las cadenas globales de producción", del BID, publicado en "Can Mexico set up in the aerospace and the software value chains as a high value added player?" Interamerican Development Bank, Integration and Trade Sector. IDD-WP- 372, 2002. Estamos en deuda con Juan Blyde, Tim Sturgeon, Sergio Carrera y Claudia Schatan por sus valiosos comentarios en distintas etapas. Agradecemos el apoyo, para continuar esta investigación, de los fondos del programa de la UNAM: IN305914: Cadenas globales de valor: retos de la política industrial.

** Profesora de tiempo completo en el Posgrado de Economía de la UNAM. Correo electrónico: brown@unam.mx.

*** Profesora de tiempo completo en el Posgrado de Economía de la UNAM. Correo electrónico: ldv@unam.mx.
} 


\section{INTRODUCCIÓN}

En los últimos treinta años, la fragmentación de los procesos productivos de las empresas transnacionales (ETN), que separan y recombinan los componentes en un sistema (Schilling, 2000) se han intensificado. Según algunos estudiosos, ha sido el resultado de los avances en la codificación del conocimiento y la estandarización de interfases entre distintas etapas de la producción con mejores estándares técnicos y de diseño (Sánchez y Collins, 2001). Así, las ETN han disminuido la integración vertical entre matriz y subsidiarias en sus actividades extraterritoriales (offshoring) para recurrir al aprovisionamiento externo conocido en la literatura de distintos países como outsourcing o subcontratación.

Esta fragmentación ${ }^{1}$ o segmentación ha permitido a las ETN especializarse en capacidades esenciales, como son el diseño, la mercadotecnia, el desarrollo de producto de componentes y los servicios tecnológicos avanzados, mientras que las empresas subsidiarias y locales de las economías semindustrializadas producen componentes o completan procesos con bajo valor agregado. Las empresas ya no compiten en su esfera nacional, sino en un mercado global; por ende, el análisis tradicional de la organización industrial tiene que evolucionar con el fin de capturar plenamente la naturaleza de las cadenas de valor globales (CVG).

La subcontratación (outsourcing) está configurada por una red de proveedores en varios países. El cambio en las tecnologías de la información y la comunicación (TIC) ha facilitado la gestión de estas operaciones crecientemente complejas, a la vez que el uso de esas tecnologías está ahora al alcance de los países emergentes. Las CVG están integradas por cientos o miles de transacciones en todo el mundo y por ello es necesario comprender la capacidad que tiene una empresa para gestionar información compleja, transferir tecnología y controlar productores.

Lejos de la creencia que identifica a la manufactura como principal demandante de servicios extraterritoriales (offshore services), la evidencia muestra que las empresas del sector terciario también utilizan la subcontratación. De acuerdo con Stephenson (2012), la manufactura sólo capta 20\% del total de los servicios extraterritoriales en el mundo, en tanto que los servicios participan con $80 \%$. Esto ha sido correctamente interpretado por el autor como la desintegración del proceso productivo no sólo en tareas que involucran bienes, sino, de

\footnotetext{
${ }^{1}$ Esta palabra se equipara con la inglesa modularization, que no tiene equivalente en español.
} 
manera importante, servicios. La industria de las TIC es también objeto de subcontratación, así como parte de los sistemas administrativos de las empresas; de hecho, según la misma fuente, el software y las industrias de Internet se encuentran entre las de mayor crecimiento junto con las industrias de cómputo, electrónica y los servicios médicos.

Es importante mencionar de nuevo que estas tecnologías están hoy al alcance de los países emergentes. Así, la subcontratación incluye una amplia gama de actividades intensivas en conocimiento que alguna vez se consideraron como del estricto dominio de los países industrializados y que ahora son desarrolladas por países en crecimiento, como señalan Fernandez-Stark, Bamber y Gereffi (2011).

El software constituye una parte importante de la cadena de valor global de las TI. que incluye el hardware para computación y telecomunicaciones, así como los servicios de telecomunicaciones y de información. La interrelación del software y los servicios de TI ha sido creciente, pues éstos incluyen planeación, integración, implantación, operación, soporte y mantenimiento de sistemas de cómputo y de telecomunicaciones. Las actividades de planeación que desarrollan los usuarios finales de estos sistemas pueden requerir los servicios de consultoría, diseño e ingeniería. La integración e implantación van juntas, debido a que la empresa proveedora de hardware es la responsable de arrancar el sistema de las TI, lo cual puede requerir la coordinación de varios proveedores de aplicaciones de software y la correspondiente adecuación a las necesidades de la empresa. Si bien, tradicionalmente, la operación ha descansado en las áreas de sistemas de las empresas, cada vez es más común que ahora la desarrollen empresas externas contratadas para el caso o bien mediante los servicios de computación en la nube. La subcontratación puede incluir manejo de equipo, operación de centros de datos y la aplicación y servicios de monitoreo, así como la gestión a distancia, incluida la seguridad (Lall, 2000; Leamer y Storper, 2001; Hualde y Mochi, 2008; Zermeño, 2011).

India es pionera en servicios y empresas de software con actividades extraterritoriales. En efecto, las empresas indias comenzaron a concentrarse en la programación de software, con la mayoría de sus trabajadores en condiciones de subcontratación (body-shopped) en Estados Unidos y Europa, de acuerdo con Dossani y Kenney (2006b). Al mismo tiempo, las ETN establecen pequeñas subsidiarias para la codificación de software y servicios de procesamiento de transacciones conforme a su estrategia de contratar mano de obra barata. Los servicios extraterritoriales se han hecho más complejos en la medida en que las 
empresas indias se han incorporado a los servicios de TI como integradoras de servicios o como diseñadoras de circuitos integrados.

A la fecha, otras economías emergentes también han ingresado a este mercado; como resultado, hay fuerzas poderosas que empujan hacia la dispersión geográfica y son un aspecto que caracteriza al mercado de software. No obstante, dichas fuerzas coexisten con otras que tienden a la concentración geográfica en el sector, debido a que las empresas - al igual que otras en los sectores intensivos en conocimiento- tienden a agruparse en ciudades o regiones específicas, como Silicon Valley, en California; la Route 128, en Massachusetts, o Dublín o Bangalore. Y en México, en ciudades como Guadalajara y estados como Nuevo León. Las empresas del primer conglomerado (cluster) interactúan con el de Bangalore y Taiwán, mientras que las de la Ruta 128 lo hacen con los conglomerados irlandés (en Dublín) e israelí (Saxenian y Hsu, 2001). Por tanto, la industria del software se caracteriza por su dispersión planetaria y su concentración en torno a ciudades y regiones específicas (Wibe y Narula, 2004; Arora y Gambardella, 2004; Heeks y Nicholson, 2002).

Este estudio busca identificar el proceso por el cual las empresas de software se han podido insertar en la cadena de las TI y los obstáculos a los que se enfrentan una vez que ya están inmersas en ella. Mediante estudios de caso, se analiza la estrategia, logros y obstáculos de estas empresas con la conjetura de que la oportunidad internacional se combinó con la utilización de apoyos de política creados ex profeso para este sector y con la presencia del espíritu empresarial de sus dirigentes. Finalmente, se investigan los logros y limitaciones de las políticas públicas como herramientas que facilitan tanto el ingreso de las subsidiarias de las ETN como la creación de las condiciones necesarias para que las empresas locales accedan y asciendan en la cadena de valor.

Después de presentar los antecedentes de esta cadena en la primera sección, se presentan las características de la CVG del software y servicios de TI en México, en la segunda, y en la tercera parte un panorama de los programas de gobierno. Los estudios de caso se analizan en la cuarta, para, finalmente, presentar las conclusiones en las que se señalan algunas recomendaciones de política económica.

\section{Algunos antecedentes de la Cadena de valor del Software}

El software constituye una parte importante de la CVG de las TI, que incluye el hardware para computación y telecomunicaciones, y los servicios de telecomuni- 
caciones y TI. La producción del software puede clasificarse en las siguientes categorías:

1) El software como producto, es decir, los programas empaquetados desarrollados para el mercado masivo, que los distribuidores venden como paquetería o bien distribuye como freeware. Esto incluye sistemas operativos, herramientas y aplicaciones.

2) El software incorporado a productos (embedded software) como autos, teléfonos, equipos de audio, robots, electrodomésticos, juguetes, sistemas de seguridad, marcapasos, aparatos de televisión, relojes digitales o aeroplanos.

3) Las soluciones de software personalizadas, esto es, las generadas por el área de sistemas de una empresa en sus instalaciones o bien por un tercero ajeno a la planta, usualmente un contratista (empresa de software). Por ejemplo, las aplicaciones para la planificación de recursos empresariales (ERP, siglas de enterprise resource planning) y las de control de clientes (CRM, por customer relationship management). La venta de estas aplicaciones se hace como productos listos para usarse (plug-and-play), aunque requieren de cierta adecuación para responder a las necesidades y requerimientos específicos del usuario final (López, Ramos y Torre, 2009).

4) El software en la nube, también conocido como cloud computing, o sea el suministro de procesamiento de información (computing) como un servicio, más que como un producto, a fin de proporcionar recursos compartidos, programas e información que se envían a las computadoras u otros dispositivos, de manera similar a servicio público (como la energía eléctrica) a través de una red (comúnmente la de Internet).

El diseño en segmentos es una característica esencial del software que se vende como producto estandarizado. Quienes lo ofrecen en paquete estructuran sus productos en familias de componentes, las cuales se desarrollan y mantienen a partir de líneas de producto o suites de software para lograr la máxima integración (Schilling, 2000). Ello hace posible cambiar o agregar segmentos de software al mismo tiempo que mantener la supervisión en una sola base de datos centralizada. Un ejemplo de esto es el System Analysis and Program Development (SAP), que fue creado como un programa ERP al que a lo largo del tiempo se le han agregado más funciones en las áreas de finanzas (CRM) y administración de recursos humanos (HRM, por human resource management). Este proceso dio origen al surgi- 
miento de las fábricas de software, que sistemáticamente capturan conocimiento referente a la producción de unidades de una familia de productos específica. Estas empresas ponen a disposición dichas unidades, en forma de activos, como patrones, marcos, modelos y herramientas, que se usan para el desarrollo de las unidades pertenecientes a la familia en cuestión, reduciendo costos y tiempo, además de mejorar la calidad del producto, todo en un mismo proceso (Greenfield y Short, 2003). El software personalizado, por otra parte, responde al hecho de que buena parte del mismo no se adecúa a las necesidades específicas de las empresas, por lo que hay una vertiente de negocio importante bajo este renglón.

Las principales naciones exportadoras de software pueden dividirse en varios grupos. El de mayor nivel lo encabeza Estados Unidos, con 63 empresas; seguido de Francia, con 60; Reino Unido, con cuatro; Alemania, con tres, y Suecia y Finlandia, con una, cada una (Software Top 100, 2011)². Los miembros más recientes de este grupo son tres célebres casos: Israel (Kaplan, 1998), India (Moitra, 2001) e Irlanda. Ello se confirma con la presencia de una o dos empresas en el informe de las cien principales empresas de 2011.

Las naciones en el segundo nivel son Canadá y Corea del Sur, con varias empresas entre las cien más importantes y que experimentan el mayor crecimiento entre las de software en años recientes. En este nivel también se encuentran las naciones en transición y que son exportadoras de software, esto es, China (Ju, 2001) y Rusia (Terekhov, 2001; Makarov, 2003)

En el tercer nivel se encuentran algunos países emergentes con que poseen una industria exportadora de software considerable (la mayor parte de ella en un rango de USD 25-200 millones). Asimismo, cuentan con uno o más conglomerados geográficos con pymes exitosas y un número limitado de países en este nivel también cuenta con algunas empresas grandes. Los países más importantes ubicados en este grupo son Brasil, México y las Filipinas. En el cuarto nivel se encuentran los países que están en una etapa incipiente como exportadores de

\footnotetext{
${ }^{2}$ Las empresas de software más importantes en el mundo generaron más de USD 220000 millones por concepto de venta de software en 2010 (Software Top 100, 2011). De ese monto enorme, $60 \%$ correspondió tan sólo a las diez principales empresas: Microsoft, IBM, Oracle, SAP, Ericsson, Nintendo, HP, Symantec, Nokia-Siemens Network y Activision Blizzard. Microsoft es, por mucho, la más grande. Las recién llegadas al club de las principales cien, en 2010, fueron: Informatica, Tibco, Emerson, Blackboard, Micro Focus y Constellation Software. Entre las desaparecidas se encuentran Microsystems y Sybase, después que las adquirieron Oracle y SAP respectivamente. La competencia entre las gigantes de la tecnología se ha incrementado en la medida en que Microsoft, Google y Apple comenzaron a desafiarse mutuamente al ingresar en territorios tradicionalmente pertenecientes a una u otra empresa.
} 
software, como Cuba, El Salvador, Jordania, Egipto, Bangladesh, Vietnam, Indonesia e Irán.

Entre los factores que explican el éxito de India, Irlanda e Israel como exportadores están la demanda mundial, así como la oferta excedente de capital humano en los años ochenta y noventa, particularmente la de personal titulado en ingeniería y tecnología (Arora y Gambardella, 2004; Heeks y Nicholson, 2002). Además, el papel que desempeñaron ciertas ETN que se reubicaron para allegarse diferentes recursos.

Por ejemplo, algunas empresas se establecieron en Israel para realizar investigación y desarrollo; otras lo hicieron en India buscando fuerza de trabajo calificada y de bajo costo, mientras que algunas más migraron a Dublín para aprovechar los incentivos fiscales y obtener acceso al mercado europeo (Arora y Gambardella, 2004). Todos los países exportadores de software de los tres primeros niveles contaron con una estrategia nacional para promover tanto a sus industrias del ramo, en general, como, en particular, a las exportadoras (Heeks y Nicholson, 2002). En este sentido, los incentivos de los gobiernos han sido importantes como estrategia activa para promover tanto un sector de tecnología de punta como también una industria del software. Etiquetadas de distintas formas: política industrial, política en ciencia y tecnología o política para la innovación (Salmenkaita y Salo, 2002), todas permitieron canalizar recursos nacionales hacia los sectores que los gobiernos consideraron de importancia estratégica para el futuro crecimiento económico, como infraestructura, parques industriales y tecnológicos y telecomunicaciones.

\section{CARACTERÍSTICAS DE LA CVG DEL SOFTWARE Y SERVICIOS DE TI EN México}

A partir de 2003, la industria del software en México creció significativamente con resultados que, de acuerdo con los pronósticos de Select Estrategia, SC (empresa que recopila datos sobre las TIC y que proporcionó información para este estudio), alcanzarán los USD 3008 millones en 2014 y un crecimiento anual promedio de $11 \%$ para el periodo 2000-2014. Hay empresas mexicanas de desarrollo de productos de software o aplicaciones para móvil o consolas de juegos que se venden directamente al consumidor final, pero la mayoría es parte de la cadena de outsourcing, ya sea de tecnología de información, procesos de negocio (BPO, por business process outsourcing) o de conocimiento (KPO por knowledge process outsourcing), tal como lo describen Gereffi, Humphrey Stur- 
geon (2005). Se puede mencionar la consultoría de negocios en TI, desarrollo y aplicaciones de TI o infraestructura de TI. Con el BPO, las empresas ofrecen servicios de administración de recursos materiales, administración de recursos humanos y administración de relaciones con el cliente, por ejemplo, los centros de contacto. El BPO puede desarrollarse para una industria específica en forma vertical (banca, industria, etc.). Por su parte, el KPO comprende innovación, diseño y pruebas, así como consultoría de negocios, legal o financiera. También puede desarrollarse para sectores específicos (salud, automotriz). México es anfitrión de una gran variedad de empresas transnacionales ubicadas en el Iто (information technology outsourcing), BPO y, obviamente en menor medida, aunque también las hay, en el KPO.

De las empresas cuya principal actividad se ubica en el sector de las TI, $76 \%$ son empresas de desarrollo de software, $13 \%$ se dedican a los medios interactivos, $7 \%$ son centros de atención telefónica y solamente $4 \%$ constituyen empresas de BPO. Sin embargo, el empleo se concentra principalmente en estas últimas (44\%); a continuación vienen las de desarrollo de software (35\%), mientras que, los centros de atención telefónica (21\%) ocupan el tercer lugar; las empresas de medios interactivos emplean menos de $1 \%$ de los trabajadores del sector. De igual manera, los ingresos los capta principalmente el subsector de BPO (68\% de los ingresos de todas las empresas de TI en 2008), mientras que las empresas de desarrollo de software se hicieron con $29 \%$ de los ingresos totales (Secretaría de Economía, 2012).

Las empresas de software integrado, al igual que las de servicios en la nube, siguen siendo algo nuevo en México y, por ello, resulta difícil obtener datos confiables de su producción y ventas. La participación del software como producto equivale a $80 \%$ de esa industria en México, aunque en su mayoría se importa. Las soluciones de software personalizadas equivalen al restante $20 \%$ del mercado, pero en ellas participan tanto grandes empresas internacionales como pequeñas compañías.

Los ingresos por concepto de productos de software personalizado han crecido a un promedio anual de $8 \%$ entre 2000 y 2014, al pasar de usd 148 millones a usd 428 millones, como se ilustra en el cuadro 1. En 2004, cuando se hizo el primer desembolso de los fondos del Prosoft, ${ }^{3}$ la tasa de crecimiento anual fue superior al promedio del resto de la economía, con $12.05 \%$ en el caso del soft-

\footnotetext{
${ }^{3}$ Prosoft es el programa gubernamental dirigido a la industria del software, como veremos enseguida.
} 
ware personalizado y $12 \%$ en el del empaquetado. La participación del software en el total de servicios de TI creció de $43 \%$ a $50 \%$.

Los servicios de TI también se han incrementado con tasas muy aceleradas (un incremento de $9.5 \%$ para todo el periodo y de $10 \%$ para el de 2004 2011). Estos servicios se suministran por proveedores internos o mediante subcontratación. De acuerdo con la entrevista realizada en Select, el costo de los servicios internos de TI es $20 \%$ más alto en contraste con su subcontratación. Hay una creciente tendencia al outsourcing de los servicios de ti y/o bien de procesamiento en la nube.

\section{Cuadro 1. El mercado del software \\ (millones de dolares de EU)}

\begin{tabular}{|c|c|c|c|c|c|c|c|c|c|c|c|c|}
\hline Concepto & 2000 & 2002 & 2003 & 2005 & 2007 & 2008 & 2010 & 2011 & 2012 & 2013 & $2014^{*}$ & $\begin{array}{c}\text { Tasa de } \\
\text { crecimiento } \\
\text { (\%) }\end{array}$ \\
\hline $\begin{array}{c}\text { Software } \\
\text { como } \\
\text { producto }\end{array}$ & 606 & 671 & 665 & 816 & 1215 & 1328 & 1376 & 1803 & 2065 & 2332 & 2580 & 10.9 \\
\hline $\begin{array}{c}\text { Software } \\
\text { a la } \\
\text { medida }\end{array}$ & 148 & 161 & 116 & 202 & 289 & 296 & 346 & 314 & 340 & 387 & 428 & 7.9 \\
\hline $\begin{array}{c}\text { Total } \\
\text { software }\end{array}$ & 754 & 832 & 781 & 1018 & 1504 & 1624 & 1722 & 2117 & 2405 & 2719 & 3008 & 10.4 \\
\hline $\begin{array}{c}\text { Servicios } \\
\text { TI }\end{array}$ & 1625 & 1768 & 1908 & 2152 & 2927 & 2989 & 3296 & 4000 & 4601 & 5204 & 5802 & 9.5 \\
\hline $\begin{array}{c}\text { Total } \\
\text { servicios } \\
\text { TI }\end{array}$ & 1774 & 1928 & 2024 & 2354 & 3216 & 3285 & 3642 & 4314 & 4892 & 5506 & 6096 & 9.2 \\
\hline
\end{tabular}

Fuente: Select Estrategia, sc.

Para el año de 2014 la subcontratación constituye el 42\% del mercado de los servicios de IT. La subcontratación puede incluir operación del centro de datos y aplicaciones, monitoreo y gestión remota de sistemas. La producción de páginas electrónicas, análisis de bases de datos, pruebas y servicios de mercadotecnia son otras actividades que se gpueden subcontratar. Finalmente, los servicios de colocación de fuerza de trabajo han adquirido creciente importancia entre las grandes empresas de software, aunque esta actividad es la de menor valor agregado. Le siguen en importancia otros servicios de TI como integración, soporte técnico y desarrollo de software. La distribución de servicios de TI puede observarse en la gráfica 1 . 
Gráfica 1. Servicios de $\mathrm{TI}$ (porcentaje del mercado, 2014)

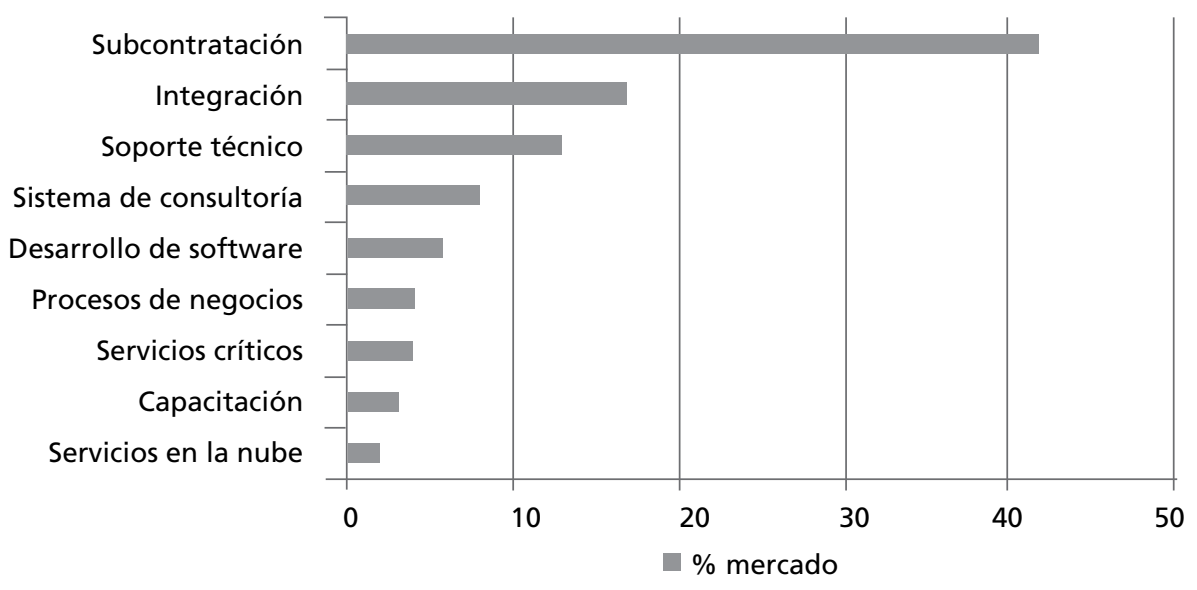

Fuente: Select Estrategia, sc.

La industria mexicana de TI está integrada por muchas empresas pequeñas y un segmento de grandes empresas que se pueden clasificar en varios grupos. De acuerdo con datos recopilados por el Banco Nacional de Comercio Exterior (Mochi y Hualde, 2009), para finales de 1999, en México funcionaban 257 empresas de programación. Únicamente 15 de ellas realizaban operaciones internacionales. En 2002, había 2098 empresas con 269620 empleados y para 2010 estas cifras se incrementaron, al pasar a 2785 empresas y cerca de 500000 trabajadores. Con excepción de un puñado de compañías, la mayor parte son pymes (esto es, con 50 a 150 empleados y ventas menores a un millón de dólares estadounidenses anuales). En una encuesta reciente aplicada a 42 empresas (Mejía, Ania y Gamboa, 2006), que equivalen a $75 \%$ del total de la industria en términos de ingreso, se muestra que son extremadamente jóvenes y, asimismo, que la productividad está relacionada con el tamaño, pues la más baja se localiza en las empresas con ventas menores a USD 10 millones anuales. El resultado es una estructura de mercado relativamente concentrada: las ventas de $40 \%$ de las empresas equivalen a $95 \%$ de las totales de esta muestra.

Los principales actores en el sector del software como producto son empresas mundiales, como Microsoft, Oracle, SAP, HP, IBM, Accenture, GapGemini y EDS, que venden al mercado nacional y operan instalaciones extraterritoriales para proporcionar servicios personalizados a sus clientes en los países desarro- 
1lados. De acuerdo con Mullan, Kenney y Dossani (2008), una segunda categoría de empresas extranjeras en México incluye algunas de la India encabezadas por las gigantes TCS, Infosys y Wipro-that, que están ampliando su presencia mundial al competir con mejores condiciones frente a las compañías de servicios en los países en desarrollo. Una tercera categoría la constituyen las empresas de TI mexicanas independientes, como Softtek, Neoris y TelmexiT, que sustituyó a Hildebrando.

Si bien hay una oferta significativa de estas empresas, tanto en cantidad como en calidad, en el Distrito Federal, Jalisco y Nuevo León, otras regiones se han quedado rezagadas. Como indican Mullan, Kenney y Dossani (2008), la concentración de actividades de TI en la Ciudad de México no refleja necesariamente ninguna estrategia de promoción específica o conglomerado sinérgico; más bien parece reflejar el tamaño e importancia de la actividad económica en dicha metrópoli. Guadalajara es el caso más exitoso de servicios de TI, ya que si bien representa un porcentaje muy pequeño de estas empresas en el país sus ingresos son mayores en comparación con los de Monterrey, que cuenta con un mayor número de compañías. Dicho éxito combina la presencia de grandes ETN, como IBM y HP —ubicadas en aquella ciudad- y un eficiente desempeño del Prosoft, como lo confirmaran entrevistas realizadas en el estado de Jalisco.

\section{Programas de gobierno para la industria}

Durante 2001, el gobierno mexicano decretó la importancia estratégica de la industria nacional del software en términos de su potencial para el desarrollo económico del país.

En octubre de 2002, se lanzó la iniciativa de Prosoft, con el objetivo de incrementar la competitividad mediante el fortalecimiento del sector de TI mexicano. En un inicio, se enfocó en la industria del software; sin embargo, a partir de 2004 se ha ampliado para cubrir todo el sector de TI. El Prosoft constituye parte de una política general que busca reemplazar el ensamblado, intensivo en fuerza de trabajo, por los servicios de alto valor agregado.

Este fondo inició sus operaciones en 2004 con un fondeo del gobierno que ascendió a USD 12.8 millones. La cifra se incrementó en 2008 al pasar a USD 65 millones, lo que movilizó recursos por encima de USD 180 millones. El Prosoft aporta $25 \%$ de la inversión total comprometida por las entidades federativas y las empresas (García, 2011). Esta iniciativa asigna fondos a una diversidad de actividades que pasan por educación y formación de recursos humanos, innova- 
ción tecnológica y desarrollo, capacidad y calidad de procesos y proyectos productivos, lo mismo que promoción y mercadotecnia, entre otras actividades.

El programa se promovió en cámaras industriales, empresas y gobiernos locales. Algunos estados de la República Mexicana han formulado políticas industriales significativas para el fomento de las empresas de TI, siendo los de Nuevo León y Jalisco los más exitosos. La importancia que los gobiernos locales han otorgado a la industria de las TI determina la eficacia con la cual se aplica la política y la cantidad de fondos que se le asignan. Como mencionamos, el estado de Jalisco es un buen ejemplo de ello.

En particular, se ha hecho un esfuerzo para asignar financiamiento a proyectos dirigidos a hacer trámites ante empresas certificadoras. La solicitud para recibir una certificación en particular puede requerir tiempo, así como capacitación y consultoría que son onerosas; de ahí que los solicitantes tengan que destinar mayores recursos. Debido al alto costo de proceso, el Prosoft desarrolló una norma de certificación ad hoc (MoProsoft) que puede resultar de mucha utilidad para las pequeñas empresas que generalmente no cuentan con los recursos necesarios.

Se han hecho avances importantes en la certificación de empresas tecnológicas. De acuerdo con entrevista a una fuente oficial en el Banco Nacional de Comercio Exterior (BANCOMEXT), en 1999 sólo cinco empresas obtuvieron una certificación Iso 9000 y un número igual contaban con la certificación CMM ( $c a-$ pability maturity model). Para 2010, en México había setenta empresas con personal certificado con CMM, lo que ubica al país en el lugar número ocho en la escala mundial, junto con Francia. En relación con otras certificaciones, como la PSP (personal software process) y TSP (team software process) o MoProsoft, un entrevistado en la Cámara Nacional de la Industria Electrónica, Telecomunicaciones y Tecnologías de la Información (CANIETI) estimó que unas 350 empresas contaban con las certificaciones mencionadas.

Mexicofirst es otra iniciativa encabezada conjuntamente por Prosoft y el Banco Mundial. Los fondos provenientes de esta iniciativa están dirigidos a costos de capacitación y certificación. En este marco, las empresas pueden recibir hasta $60 \%$ del costo total del proyecto. Por su parte, IT LINK es una iniciativa que se originó en Singapur con para certificar los procesos de negocio de las empresas con el fin de ayudarlas a buscar clientes entre las ETN. Esta iniciativa reúne información mediante una sólida red operativa que permite que las empresas respondan a compromisos mediante una alianza o asociación de proveedores. A través de IT LINK, las empresas asociadas pueden fácilmente estar en contacto con una vasta red de clientes potenciales. 
En el periodo de 2000 a 2012, el número de empresas certificadas creció notablemente, pues de sólo cuatro pasó a 526 (setenta en CMMI), es decir, una tasa de incremento media anual de $58 \%$.

Algo destacable del Prosoft es que ha evolucionado para atender nuevas orientaciones de la industria de TI. Más recientemente, a través de este programa, y con apoyo del Consejo Nacional de Ciencia y Tecnología (CONACYT) y Pro México, se están destinando recursos a la Ciudad Creativa Digital para apoyar la formación de un conglomerado de empresas de medios interactivos en el centro de la ciudad de Guadalajara.

Además de brindar espacios e infraestructura para esas empresas, el parque integrará espacios y tecnologías disponibles que permitirán a la ciudadanía experimentar con éstas en tiempo real y probar productos y servicios, particularmente de sistemas de diseño y gestión urbanos. A la par de estos programas específicos, es importante mencionar que las empresas tienen acceso a los estímulos a la innovación del CONACYT y otros programas para las pymes.

Por último, cabe hacer referencia a los programas de la Fundación México-Estados Unidos para la Ciencia, que opera como una oficina de consultoría gerencial que proporciona a las empresas una estructura operativa sólida para que se posicionen en una CVG (Techpyme) y una altamente especializada aceleradora de empresas (TechBA). Los servicios de TechBA reciben financiamiento del programa de la Secretaría de Economía enfocado a las pymes. Inicialmente, TechBA se enfocó en las empresas posicionadas en el mercado de tecnología de punta; sin embargo, en años recientes se ha especializado en cadenas de valor específicas, dentro de las cuales está la de las TI. En este rubro han apoyado aproximadamente a noventa empresas, es decir, $25 \%$ del total que ha atendido. Cuenta con ocho oficinas en distintos estados de Estados Unidos y una en Europa. Tres de ellas están muy relacionados con la industria de las TI: Silicon Valley, en California; Austin, en Texas, y Seattle, en el estado de Washington.

Algunos investigadores han identificado debilidades estructurales en las empresas de TI. Mullan, Kenney y Dossani (2008) señalan como un obstáculo muy importante la ausencia de economías de escala en las empresas mexicanas en comparación una a una con sus homólogas en la India. En efecto, en este país las empresas grandes cuentan con más de 30000 empleados (algunas de ellas incluso llegan a 100 000), con numerosas empresas medias de nicho (5 000-29 999 empleados) y muchas más empresas pequeñas (menos de 5000 ).

En México, otra limitante es la ausencia de especialización de mercado, lo cual sería de esperar dado el pequeño número de empresas. Así, es muy co- 
mún que las empresas de esta industria se enfoquen en más de un servicio. Mejía, Ania y Gamboa (2006) encontraron que tan sólo 30\% de las empresas encuestadas tenían un servicio que equivalía a por lo menos $50 \%$ de su negocio, pero señalan que en algunos de estos casos se debía a que proporcionaban servicios a un solo cliente muy importante.

Tomando a las empresas extraterritoriales indias de TI como punto de referencia, Mullan, Kenney y Dossani (2008) identifican un enorme potencial de las empresas mexicanas debido a: 1) una enorme ventaja por su ubicación geográfica, cuando se las compara con las de Asia e, incluso, otras de América Latina, ya que la proximidad al mercado estadounidense facilita significativamente la interacción; 2) costos de telecomunicaciones más bajos en comparación con los de India, y 3) la superioridad de la infraestructura con que cuenta México en comparación con la de India, que presenta un déficit en términos de aeropuertos, transporte público y hoteles, todo lo cual es un obstáculo obvio para hacer negocios. Aun cuando los salarios en India son más bajos, sus trabajadores reciben un pago extra para compensar los gastos de transporte, gasto que las empresas mexicanas de software no tienen.

No obstante, estas ventajas por sí mismas no son suficientes para que las empresas de TI mexicanas compitan con sus pares indias. Por ejemplo, los costos de las telecomunicaciones pueden ser un gasto significativo para los call centers, que requieren de un ancho de banda significativo, aunque estos costos no sean necesariamente los más fuertes frente a los que incurren las empresas de TI. Como es sabido, el costo de telefonía celular y de suscripción a la banda ancha (fija y móvil) es elevado en México, si se le compara con la gran mayoría de los países de la Organización para la Cooperación y el Desarrollo Económicos, lo que se agrava con la deficiente calidad del servicio (Schatan y Enríquez, 2012).

Más importante es que en México la demanda para la carrera de ingeniería se ha estancado o incluso decrecido, mientras que en India, por el contrario, ha habido un incremento impresionante de ingenieros $\mathrm{y}$, por tanto, en ese país se ha logrado conformar un grupo significativamente mayor de ellos, con un mejor dominio del inglés y que en su mayoría cuentan con una visa $\mathrm{H}-1 \mathrm{H}$ porque han emigrado, vivido por un tiempo o estudiado previamente en Estados Unidos. Esto explica el tamaño enorme y la competitividad de las empresas indias y también las dificultades que las grandes empresas incluidas en las entrevistas realizadas tienen para reclutar fuerza de trabajo calificada en las cantidades que requieren.

Una última desventaja puede considerarse el relativamente bajo porcentaje de personas que usan Internet, que es un factor de demanda para la industria 
de TI: $38 \%$ en China, en contraste con $36 \%$ en México, a pesar de que el ingreso per cápita de los mexicanos es casi el doble que en el país asiático. En Brasil, esta cifra es de $45 \%$, no obstante que su ingreso per cápita también es menor que el de México (Schatan y Enríquez, 2012).

\section{LA CADENA DE VALOR DEL SOFTWARE: ESTUDIOS DE CASO}

Antes de entrar de lleno en esta sección, conviene aclarar que los criterios para escoger estos casos fueron los de empresas con reconocidos logros, recomendadas por miembros de la CANIETI, el Instituto Jalisciense de Tecnologías de la Información (IJALTI) o el Consejo del Software en Monterrey. Es decir, son casos de los que se pueden derivar lecciones. Buscamos cubrir empresas de distintos ramos dentro de las TI: subcontratación de servicios de TI y de negocios (Softtek), capacitación especializada en TI en la nube (Scio), capacitación especializada en negocios de TI y certificación (Qualtop), desarrollo de software para los sectores energético, salud y financiero (Delaware), sistema integral de TI para la gestión hospitalaria (Medisist) y juegos, medios interactivos y animación (Kaxan). Obviamente, al escoger sólo seis, dejamos fuera a muchas empresas igualmente valiosas. Tal como se ha señalado en otros trabajos, no se trata de hacer una historia de éxitos, sino de la senda cuesta arriba con muchos altibajos y obstáculos en el futuro.

El método utilizado fue de entrevistas a profundidad, guiadas por algunos puntos generales, entre los cuales están el surgimiento de la empresa, las características de su trayectoria, la formación de capacidades del equipo y las motivaciones e incentivos para orientarse como lo hicieron. Asimismo interacción con empresas a lo largo de la cadena, experiencias con la utilización de apoyos de los programas gubernamentales y opiniones al respecto, visión de la empresa misma frente a sus competidores. En pocas palabras, las fuerzas motoras y los obstáculos en distintos nichos del mercado en la CVG.

\section{Softtek (servicios near-shore)}

Tomando en consideración que India es, por mucho, el principal proveedor de servicios de TI entre los países emergentes, el caso de Softtek muestra que hay distintas fórmulas y opciones que permiten el ingreso de los países emergentes a la CVG de servicios de TI. A la fecha, Softtek es una empresa proveedora mundial de servicios de TI, con cerca de 6400 asociados en 33 oficinas ubicadas en Amé- 
rica del Norte, América Latina, Europa y Asia. Es la empresa mexicana más grande de software, con tasas de crecimiento aceleradas y un portafolio de servicios bien diversificado. La empresa la constituyeron, en 1982, tres ingenieros que habían laborado en la división de sistemas del Grupo Monterrey. Posteriormente se unieron a la empresa dos socios más. El producto inicial fue el desarrollo de software para empresas mexicanas grandes y medianas, no obstante, el portafolio de la empresa ha evolucionado sustancialmente.

En un inicio Softtek, se concentró en servicios relacionados con aplicaciones, incluyendo desarrollo de ellas, pruebas de software, manejo de aplicaciones y servicios de seguridad para éstas, así como SAP e inteligencia de negocio. De acuerdo con el entrevistado, a la fecha, el desarrollo de aplicaciones representa la participación más grande en los ingresos de Softtek. Esta empresa desarrolló el Softtek's Software Development Process (SSDP ${ }^{\circledR}$ ), una metodología de cumplimiento de fases y objetivos que constituye la base subyacente de los servicios de desarrollo de aplicaciones que brindan. Otros de sus servicios incluyen soporte de TI (servicios de soporte a usuarios, servidores y servicios de datacom, y soporte de procesos para empresas en TI), BPO (servicios de adquisiciones) y SAP y licencia de software de inteligencia de negocio (SAP, Informática, IBM Cognos).

Los destinos de exportación iniciales de la empresa a comienzos de los años noventa incluyeron Perú, Brasil, Argentina y Colombia. No fue sino hasta 1997 que lanzaron un programa de exportación ambicioso hacia el mercado de Estados Unidos. De acuerdo con el entrevistado, las razones que se encuentran detrás de esta estrategia fueron las siguientes: primero, en la empresa se percataron de que si se quedaban en el mercado nacional no habría suficiente espacio para crecer $\mathrm{y}$, por tanto, no podrían diversificar adecuadamente el riesgo; segundo, vieron el problema del $\mathrm{Y} 2 \mathrm{~K}$ como una ventana de oportunidad en el mercado estadounidense $\mathrm{y}$, lo más importante, comprendieron que las grandes corporaciones estaban a la búsqueda de opciones de subcontratación fuera de India o China.

Softtek, en su estrategia, ha aprovechado la proximidad con Estados Unidos como una ventaja atractiva para los clientes de ese país, los cuales tienen necesidad de soluciones rápidas para los problemas que enfrentan sus negocios y, además, la ventaja que ofrece el hecho de contar con una cultura corporativa compatible con ese país. La empresa percibió que la proximidad geográfica es de gran ayuda para el alcance de los proyectos y la evaluación de las necesidades de los clientes. Diversos aspectos — que van de estar a una dis- 
tancia que se cubre con un fácil desplazamiento a ubicarse en la misma zona horaria- mejoran sustancialmente la comunicación. Por último, pero no por ello menos importante, se dio gran importancia al beneficio que significan las visas de corto plazo - aspecto que trajo consigo el Tratado de Libre Comercio de América del Norte (TLCAN) — y la seguridad que ofreció el gobierno mexicano al comprometerse con el acuerdo sobre los Aspectos de los Derechos de Propiedad Intelectual Relacionados con el Comercio (ADPIC) de la World Intellectual Property Organization (WIPO). De manera que, aun cuando China e India mantienen un claro liderazgo, para Softtek fue evidente que había un nicho para las empresas mexicanas.

Al preguntarle sobre el tipo de capacidades que la empresa ha desarrollado para abrirse paso en el mercado de Estados Unidos, el entrevistado señaló que cuando consideraron entrar a él, la empresa ya contaba con una planta de personal de aproximadamente 1800 ingenieros certificados en tecnologías y metodologías específicas. Asimismo, la empresa contaba con un programa denominado Softtek Academy, mediante el cual se contrata empleados por seis meses, durante los cuales reciben capacitación; asimismo, el programa ofrece becas a estudiantes que cursan los últimos años de universidad. Otro programa interesante al que hizo referencia dentro de la empresa fue el TSP, que permite un mejor desempeño de proyecto, ofrecer productos de forma más expedita y con alta calidad, y mejorar el equilibrio de la vida laboral de los empleados. Además, al crear equipos autodirigidos, Softtek reportó una creciente productividad y la reducción de costos mediante resultados más predecibles. De acuerdo con el entrevistado, cerca de $15 \%$ de los asociados son líderes de proyecto y todos ellos están certificados.

En lugar de centrarse en competir directamente en precios, Softtek diferenció sus productos. Registró como marca el concepto de near-shore, el cual hace referencia a suministrar servicios en un centro adyacente o cercano. Con nueve centros de servicios a escala mundial ubicados en México, China, Brasil, Argentina y España, Softtek ofrece a sus clientes -empresas de primer nivel en más de veinte países - soluciones de negocio que les ayudan a mejorar la oportunidad de sus operaciones, reducir costos de aplicaciones existentes y suministrarles aplicaciones con mejor ingeniería probada, y producir resultados predecibles. En el marco de este plan, desde sus instalaciones en México se ofrece soporte al mercado estadounidense; desde las ubicadas en España, a empresas inglesas y francesas, mientras que desde las de Argentina se da soporte a empresas en América del Sur. 
Los ingresos por concepto de exportaciones equivalen a $75 \%$ del total de ingresos de la empresa, con un ingreso reportado de cerca de USD 250 millones. Entre los clientes de Softtek se incluyen bancos y financieras, además de otras empresas que aparecen en Fortune 500. El entrevistado destacó que muchos de los clientes de Softtek ya tenían contratados servicios con empresas de India, cuestión que lo lleva a pensar que esos clientes recurren a los servicios de Softtek como una opción complementaria, ya sea para diversificar sus proveedores o para resolver necesidades que no han solventado las contrapartes asiáticas de Softtek.

El cliente insignia de la empresa es General Electric (GE), con la cual tuvieron un contrato inicial pequeño, pero cuyas operaciones fueron incrementándose, tanto en ventas como en servicios provistos. Cuando GE Capital International Services decidió reubicar en India una gama de actividades corporativas, como tarjetas de crédito, operaciones administrativas, trabajo de call center, finanzas internas y contabilidad, GE vendió la división Ddmesis-a de GE Capital International Services America —especializada en el desarrollo, mantenimiento, gestión e integración de sistemas de software- a Softtek (Dossani y Kenney, 2006a). Esta adquisición amplió enormemente el portafolio de aplicaciones y servicios de esta última. A la fecha, Softtek tiene relaciones comerciales con varias divisiones de GE, entre ellas GE Capital y GE Manufacture.

En agosto de 2007, Softtek adquirió IT UNITED (basada en China) para expandir sus capacidades en el mercado asiático. En la actualidad, Softtek cuenta con 300 empleados en aquel país. De acuerdo con el entrevistado, la importancia estratégica de esta inversión es triple: primero, el mercado local chino ofrece enormes posibilidades de crecimiento; segundo, ciertos clientes de Softtek tienen operaciones en China y la empresa puede atenderlas desde sus nuevas instalaciones y, tercera, contar con una base en ese país ofrece la posibilidad de posicionarse en los mercados globales las 24 horas del día, los 365 días del año. En Softtek denominan a esta estrategia comofollowing the sun.

Otro mercado importante es el de Brasil, cuatro veces mayor que el mexicano. Aquel país cuenta con un número mayor de empresas medias en comparación con México y tienen mayor liquidez para invertir en servicios como los que ofrece Softtek.

No obstante todos los esfuerzos por incrementar sus capacidades, la principal barrera u obstáculo que enfrenta la empresa es la insuficiente oferta de empleados calificados que dominen el inglés. En una entrevista con Universia Knowledge@Wharton, Blanca Treviño (CEO de Softtek) describió la escalabili- 
dad como una de las limitaciones más importantes que Softtek comparte con otras grandes empresas de TI en México. Señaló que hay un umbral en la escala de operaciones al que se puede llegar. India y China pueden convocar miles de profesionales en software en poco tiempo, lo cual sería prácticamente imposible en México. No obstante, la estrategia global de la empresa aprovecha la ya citada proximidad y adaptabilidad con sus clientes en Estados Unidos y España.

Dependencias en los tres órdenes de gobierno (federal, estatal y municipal) constituyen parte del mercado nacional de Softtek. Dichas dependencias atraen contratistas en TI mediante licitaciones rigurosas, en las que compiten buscando satisfacer rigurosas especificaciones. Dichas licitaciones implican prácticamente por regla inversiones muy fuertes. Softtek subcontrata varias empresas (o viceversa) al participar en las licitaciones, debido a que es muy poco frecuente que una empresa produzca cada aspecto o servicio requerido; es el único caso donde subcontrata otras empresas.

A pregunta expresa sobre momentos de desafío, errores y lecciones aprendidas, el entrevistado señaló que el crecimiento acelerado es una bendición, si bien implica riesgos. A comienzos de 2000, Softtek recibió la asignación de varios contratos, sin embargo, no pudo cumplir satisfactoriamente con todos los servicios contratados debido a: 1) falta de personal calificado, 2) falta de capacidad para proporcionar lo ofrecido al cliente o 3) valoración insuficiente de los aspectos de rentabilidad. En Softtek se ven constantemente forzados a recordar las lecciones aprendidas, en especial en sus nuevos empeños que implican riesgos; entre dichos empeños se encuentra el software integrado y, en el futuro cercano, suministro de servicios en la nube.

En opinión del entrevistado, Prosoft ha sido exitoso debido a que sentó las bases para el desarrollo de los servicios de TI en México. Softtek ha aprovechado el programa de certificación que ofrece Mexicofirst. Mencionó la ayuda que Pro México les proporcionó en la promoción de exportaciones como un factor determinante para Softtek. También fue claro con respecto a la falta de éxito de los programas de innovación del CONACYT, los cuales se supone que están dirigidos a la creación de nuevos productos, sin embargo, la iniciativa carece de una clara comprensión del software y su potencial innovador. Señaló que, a su juicio, esta falta de comprensión explica por qué Softtek fue rechazada cuando solicitó financiamiento.

El principal problema que la actual política económica sigue sin resolver es la falta de financiamiento necesario para competir en el mercado global. Esto resulta particularmente problemático para los servicios de ті у emprendedores en 
el ámbito del software debido a que sólo pueden ofrecer garantías intangibles (es decir, capital humano y conocimiento). Ante ello, los inversionistas tradicionales y bancos son propensos a rechazar las solicitudes de financiamiento que aquéllos presentan.

\section{Scio (servicios de software en la nube)}

Como ya se mencionó, la estructura del mercado del software en México se caracteriza por contar con unos cuantos casos estelares, la mayor parte de ellos empresas extranjeras y numerosas nacionales pequeñas y medianas. Debido a su escala limitada, muchas de estas últimas restringen sus servicios al mercado nacional que, debido a su tamaño también limitado, implica una fuerte competencia. Softtek ingresó al mercado global gracias a su talento emprendedor, capacidad para hacer adquisiciones, finanzas sanas, productos de alta calidad y rentabilidad, y el suministro de una gama muy amplia de productos.

Las empresas medianas y pequeñas sólo cuentan con recursos limitados y aun si cuentan con capacidades similares tienen que encontrar un nicho donde sean competitivas para convertirse en exportadoras exitosas. Un caso evidente de ese tipo de empresas es el de Scio, una pequeña empresa mexicana con no más de cincuenta asociados que proporciona servicios a empresas de software estadounidenses, canadienses e inglesas. Un factor importante detrás del éxito de Scio es que se especializa en un área de conocimiento.

La empresa se fundó en 2003 en Morelia, Michoacán. El entrevistado, un ingeniero mexicano con grado de maestría, que obtuvo en Austin, Texas, había trabajado previamente como consultor de sistemas por cuatro años, con lo que se percató de que había una oportunidad para ofrecer en México servicios offshore a empresas que los requerían. De acuerdo con sus comentarios, aunque el primer año fue difícil, la empresa evolucionó de un negocio pequeño en su domicilio a una operación de multiservicios, en constante lucha por sobrevivir. En 2006, se enrolaron en el programa de consultoría TechвA, en Silicon Valley y, después, en Seattle. La principal recomendación de TechBa a la empresa fue la de encontrar un nicho en el cual tuviera mayor experiencia. Identificaron las tendencias en la industria y tomaron la decisión de reducir sus multiservicios para enfocarse en el software para el servicio vía Internet, también conocido como servicios en la nube.

Los servicios en la nube incluyen, entre otros, entrega de productos y servicios de software vía Internet, en lugar de vender exclusivamente software 
como producto. Scio ofrece tres servicios: 1) consultoría innovadora que ayuda a las empresas a formular una estrategia (soluciones técnicas) para iniciar operaciones en la nube, 2) programas de capacitación a personal de empresas que buscan migrar a la nube y comprender todos los cambios concomitantes en los paradigmas de negocio y 3) soluciones de software y aplicaciones vía Internet.

La capacitación se ha convertido en una prioridad de la empresa y, por ello, sus empleados han ingresado a programas públicos y privados, incluyendo varios de los segundos en ciudades como Denver, Austin y San Francisco. Estos programas con auspicio privado reciben participantes de todo tipo de empresas que desean familiarizarse con los procesos y los servicios en la nube y los problemas que implica migrar software a dicha plataforma. Un ejemplo de dichos programas, es el de Microsoft (cliente número uno de Scio, que recientemente lanzó el Windows Assure para la nube), que comprende dos etapas: el primer módulo del programa se dio en el home-based business de Richmond, Washington, mientras que el segundo lo fue en México, con la participación de treinta empresas mexicanas.

Aun cuando Microsoft es el cliente más importante de Scio, la mayor parte de sus ingresos proviene de empresas de software medianas. Únicamente $5 \%$ de sus clientes son mexicanos, pues la mayor parte de las empresas nacionales no tienen capacidad para cubrir los costos de Scio.

La empresa hace esfuerzos para consolidar su reputación en el mercado estadounidense. Al perder algunos contratos frente a firmas cuyos servicios son más caros, aunque con menor experiencia, en Scio llegaron a la conclusión de que el precio por sí mismo no les da la competitividad que necesitan. Recientemente, iniciaron operaciones en Seattle para proporcionar servicios más personalizados.

El primer obstáculo en Estados Unidos fue la falta de reconocimiento de México como un lugar destacado en el campo de los servicios de TI. Esto está cambiando poco a poco, en la medida en que muchos de los clientes de Scio ya no quieren contratar empresas indias o chinas. El segundo obstáculo fue de naturaleza financiera: su estrategia de crecimiento se basaba en el flujo de efectivo, lo que limitaba seriamente el potencial de crecimiento de la empresa. Encontraron dificultades mayores al solicitar crédito, debido a que las fuentes tradicionales argumentaron que el proyecto carecía de escalabilidad. La tercera limitante, y quizá la más importante, es la escasez de fuerza de trabajo que domine el inglés y que tenga formación en programación. 


\section{Qualtop (mejores prácticas de software)}

No es necesario exportar para formar parte de la CVG de TI, lo cual puede observarse en el caso de Qualtop. Los exportadores de servicios de TI, así como quienes proveen servicios al gobierno, deben contar con altos estándares de calidad. La competitividad de esta empresa descansa en ser una de las primeras en entender esta necesidad y en conseguir todas las certificaciones necesarias. Así, Qualtop tiene como clientes empresas transnacionales de distintos países que laboran en México y Argentina, además de compañías nacionales.

Qualtop es subsidiaria de una empresa de aplicaciones de software argentina que se inició con tres ingenieros y que, a la fecha, cuenta con más de cien consultores. La empresa quedó constituida en México en 2003, con el fin de desarrollar aplicaciones. Su modelo de arranque de negocio pronto cambió en la medida que identificó oportunidades de especialización en los procesos de optimización de software y certificaciones, por lo que dejó atrás las aplicaciones de software. Posteriormente, en 2009, crearon una nueva empresa con sede en el conglomerado de software del IJALTI, en Guadalajara, donde, de acuerdo con el entrevistado, el apoyo al emprendedor resulta particularmente eficiente. También cuentan con oficinas en la Ciudad de México, Culiacán y Tijuana, donde se encuentra la mayor parte de sus clientes corporativos.

El entrevistado narra el proceso que los llevó a identificar su nicho de mercado. La historia se remonta al equipo en Argentina, que observó altos niveles de desorden en los procesos de sus clientes, lo cual no les permitía establecer sus necesidades ni exigencias. Esto fue la oportunidad. Así, Qualtop desarrolló una herramienta que les permitió organizar procesos y, para ello, llevaron a cabo un proyecto interno para obtener la certificación CMM. Qualtop tiene cuarenta asociados y los fondos Prosoft han sido de importancia crucial para sus operaciones. Como resulta difícil reclutar consultores calificados con experiencia, tanto en desarrollo de software, como con pruebas y conocimiento de Six Sigma, la empresa recurrió inicialmente a los fondos de Prosoft para invertir en desarrollo de capacidades. El personal ha tomado varios cursos introductorios a CMMI ( $c a-$ pability maturity model integration) y el curso especial del Software Engineering Institute (SEI).

La empresa también ha utilizado el Prosoft para certificarse en CMMI3 como empresa consultora. En América Latina es la única consultora en calidad de procesos de software. El CMM se ha convertido en una certificación muy importante para que una empresa sea proveedora de software para ETN o gobiernos. 
La asesoría y capacitación de la empresa busca apoyar el updating de las empresas desde los aspectos más básicos hasta la certificación. Los clientes de Qualtop son muy diversos: algunos de ellos desarrollan exclusivamente software, otros subcontratan, mientras que algunos más tienen un área importante de sistemas. La meta es aumentar la productividad, disminuir errores y evitar un eterno proceso de pruebas por venta, después de generar un desarrollo.

Hoy en día, la empresa tiene $87 \%$ de cuota de mercado de estas certificaciones, es decir de cada cien empresas certificadas en CMM, 87 fueron sus clientes. También están asesorando para la certificación de ISO 20000 e ISO 27001, así como de TSP y PSP, aunque en menor medida.

De la misma manera en que la empresa utilizó el Prosoft en la formación de sus capacidades, el financiamiento a sus clientes pymes por medio de este programa resultó fundamental. En su opinión, el Prosoft resulta ser un buen instrumento, aunque su desempeño depende de la eficiencia de los gobiernos locales, que deben aportar $25 \%$ del total del proyecto. Las autoridades de Jalisco han entendido las ventajas de especializarse en conglomerados de software en la capital del estado y, por ello, el Consejo de Ciencia y Tecnología de Jalisco, las cámaras empresariales y las universidades trabajan para ese fin. Las iniciativas del Prosoft en Jalisco buscan simplificar los procedimientos para las pequeñas empresas, con el fin de que accedan a fondos para certificación. Este no es el caso en la Ciudad de México, ni en el estado de Michoacán, donde se ubica Scio, que no pudo aprovechar el financiamiento por medio del Prosoft. Esto hace complicado para la empresa la atención a las pymes, que constituyen aproximadamente $50 \%$ de la cartera de Qualtop. Recientemente están incursionando en los fondos del Instituto Nacional del Emprendedor para gestionar proyectos con pymes.

Los servicios que prestan a las compañías grandes constituyen el otro $50 \%$ de sus ingresos por concepto de ventas. La empresa no exporta debido a que su consultoría es interactiva y exige que los consultores visiten a sus clientes por lo menos dos veces a la semana. Un plan a futuro es instalarse en otro país.

En opinión del entrevistado, al igual que otros participantes en la industria con los que se habló, México no puede competir directamente con India, por lo que más bien tiene que desarrollar nichos con base en sus ventajas comparativas en servicios de desarrollo de software, gestión de proyectos y análisis de requerimientos. En otras palabras, no obstante el éxito de algunas empresas, son pocas las posibilidades de participar en actividades offshore (principalmente fuerza de trabajo) en gran escala, por lo cual, las actividades de software con alto valor agregado deben constituir el objetivo de las empresas mexicanas. 


\section{Delaware (capacidades tecnológicas y alta calidad)}

Delaware se funda hace 17 años por dos hermanos, en Monterrey. Su actividad principal ha sido el desarrollo de software a la medida. Inicialmente, atendían compañías mayoritariamente locales de tamaño medio. A pesar de ser una empresa pequeña, pues sólo cuenta con 22 empleados con formación profesional y algunos con posgrado, tiene presencia en el mercado nacional y puede decirse que ahora también en el internacional.

Pronto se dieron cuenta que para vender en Estados Unidos era necesaria la certificación internacional. Comenzaron por obtenerla en México. El Prosoft les concedió apoyo para un proyecto de mejoramiento de competencias de calidad y gracias a que el modelo de trabajo es muy ágil lo aplicaron en unos cuantos meses y en todo el año de 2008. Como la certificación CMMI les resultaba onerosa en términos de precio y por el nivel de recursos que implica, optaron por la certificación TSP, que obtuvieron con un alta calificación, por lo que el Software Institute los seleccionó como la primera empresa en nivel organizacional en múltiples proyectos. Esto les ha abierto muchas puertas.

Sin embargo, la certificación es apenas un primer paso. Para vender en Estados Unidos hay que ser conocido allá. En alguna ocasión no pudieron concursar por un proyecto muy factible del Departamento de Energía porque no estaban domiciliados en territorio estadounidense. Esta fue una lección sobre la importancia de estar ubicado en ambos países. La oportunidad llegó cuando una empresa les solicitó un proyecto para sus empresas en Estados Unidos con la condición de que tenían que facturar en ese país. Así, fundaron una empresa en Austin en forma virtual, por lo que hoy en día uno de los propietarios se encuentra ahí para atender la demanda en el vecino país.

Para esta empresa un paso importante ha sido definir sus capacidades, pues durante mucho tiempo atendían todo tipo de proyectos. Actualmente, se enfocan en sólo tres sectores: salud, financiero y energía, centrándose en aquellos clientes que requieren alta calidad, es decir cuyo sistema es crítico y no puede fallar. Su modelo de trabajo le permite operar con error mínimo y con certidumbre de pronta entrega: la desviación de su calendario es muy pequeña, de $4 \%$. Entre sus logros está un muy complicado trabajo para una empresa internacional de la industria eléctrica, la cual reconoció que "nunca habían tenido un sistema que no presentara una falla".

Siendo una compañía con tanta experiencia técnica, sus problemas han venido de la parte administrativa. Por ejemplo, con el cambio de la oficina se 
enfrentaron a una escasez de flujo de caja y, hay que decirlo, no sólo por falta de planeación financiera, sino por el efecto de la crisis que obligó a muchos clientes a detener sus inversiones. Afortunadamente, ya han sorteado este problema, que sin duda les dejó muchas enseñanzas. Otro problema es la dificultad de comercializar, según el entrevistado, pues esta empresa difícilmente puede ser reconocida en un mercado tan amplio, lo cual la coloca en una posición vulnerable ante la demanda. Por su dimensión, se ve limitada a tener un departamento de ventas o una persona asignada para tal propósito. Sin embargo, goza de la certificación del Software Institute y una señalización de la calidad de la empresa por parte de ellos que les es muy valiosa.

El tamaño de las empresas mexicanas de software, como se ha comentado, puede ser una limitante para acceder a grandes proyectos. Cuestionado sobre la factibilidad de acceder a un proyecto muy amplio estableciendo alianzas con otras empresas, el entrevistado manifestó sus dudas al respecto, porque para complementarse dos o tres compañías en un equipo mayor es necesario proporcionar información y no todos están dispuestos a ello o, alternativamente, cobran muy caro por trabajar por subcontrato en un proyecto. Es decir, formar consorcios de pymes para grandes proyectos encuentra muchas dificultades.

Un problema constante para la empresa ha sido la falta de capital. Si bien gozó de los beneficios del Prosoft para la primera certificación y equipamiento, tras crecer y tratar de ingresar al programa del Banco Mundial para la segunda certificación, no ha logrado obtener financiamiento bancario. Cuando lo solicitó en Hirpyme no calificaron porque acababan de tener un año malo y, aunque ya tenían varios proyectos por cobrar, eso no constituyó un indicador para el banco.

Reflexionando sobre la competencia de India, el entrevistado señala que Infosys jamás habría llegado a la posición que tiene en Estados Unidos si no hubiera ya crecido en su país con proyectos para distintas agencias gubernamentales. En cambio, en México, el gobierno otorga los mayores proyectos a las grandes empresas extranjeras. En otras palabras, no sólo hay un problema de financiamiento sino que, a diferencia de otros países competidores, en los que hay un esfuerzo consciente por crear una industria de software, en México no hay una política de apoyo a las empresas nacionales del ramo.

\section{Medisist}

La empresa se funda en diciembre de 1994, como un consorcio mexicano-chileno-colombiano dedicado al desarrollo de soluciones de TI en el sector salud, con 
expertos en informática médica latinoamericanos, estadounidenses y alemanes. Los socios originalmente realizaron un doctorado en informática médica y de ahí surge la inquietud por introducir las mejores prácticas de Alemania a sus países. En 2001, el consorcio se desintegra y queda en México como Medisist, SA de Cv. La empresa cuenta con sesenta empleados.

Inicialmente, desarrolló un sistema médico con un examen integral de expedientes clínicos que fue perfeccionándose hasta lo que tienen ahora, que es un sistema completo de gestión hospitalaria con subsistemas departamentales (radiología, laboratorio, urgencias, farmacia, banco de sangre, veterinaria y, actualmente, teleconsulta) actualizado de acuerdo con las normas internacionales. La relación con el cliente es cercana y la empresa apoya todos los sistemas de TI del hospital que los contrata y los adapta y actualiza conforme a los cambios nacionales e internacionales.

Al decir del entrevistado, desde su nacimiento, la empresa tuvo el proyecto de participar en el mercado internacional, aunque las ventas al exterior han fluctuado. Al principio, lo hizo en Sudamérica y actualmente incursiona en Canadá, España y, próximamente, en Estados Unidos. La empresa tuvo oficinas en este último país, aunque sin éxito. Sus clientes se ubican en el sector de salud privado, pero ahora están volviendo a incursionar en el sector público, en donde con anterioridad no habían tenido buena recepción.

La mayor parte de sus empleados son ingenieros y algunos médicos. La empresa cuenta con un departamento de innovación y desarrollo, la colaboración académica la realiza con asociaciones nacionales e internacionales para estimular la innovación, en tanto que el intercambio de experiencias y profesionales han sido una de las más importantes características de la empresa.

En una empresa que atiende clientes de tamaño medio a grande es muy importante la calidad. Así, se mencionó la necesidad de avanzar en una larga curva de aprendizaje. De ahí, la inquietud por capacitar o contratar personal muy especializado para proyectos específicos. El Prosoft ha sido útil para la empresa en materia certificación y equipo, ya que están certificados internacionalmente en CMMI4, lo que ha sido un logro importante.

En un negocio como el de TI, la interacción entre empresas es fundamental y por ello le han dado la debida importancia. Comercialmente, participaron en la formación de la primera integradora de empresas de TI en México (Aportia), la cual ya no existe y participan en el consorcio IP-SP (Infotec), en la CANIETI, en el conglomerado del IJALTI y en la Plataforma Tecnológica Mexicana (PTM), entre 
otras instancias. Además, tiene alianzas estratégicas con varias instituciones, como la Universidad de Zacatecas, el Cinvestav y la Universidad de Guadalajara.

En el presente, la competencia de empaquetados importados como un SAP (sistemas, aplicaciones y productos para procesamiento de datos) es fuerte, a pesar de que no están adaptados a las necesidades hospitalarias como lo estaría su producto, que puede tener mucha aceptación en los grandes hospitales privados e incluso en los públicos. Así mismo, puede suceder que hospitales pequeños compren sistemas muy limitados que serán inútiles cuando crezcan. El reto de Medisist es enfrentar esta competencia y convencer de que sus productos y servicios están a la altura en precio y calidad.

\section{Kaxan (tecnología y creatividad)}

Kaxan se fundó en 2009, con un equipo inicial de cuarenta personas, para proyectos pequeños, juegos de baja complejidad, con lo cual logró dotar de capacidades a sus miembros en las nuevas tecnologías. Conforme lograron sus primeros juegos exitosos, Nintendo les abrió las puertas para trabajar con una consola de desarrollo. Su contacto con esta compañía les ofreció otras oportunidades y desarrollaron un juego con el personaje de El Chavo para la consola Wii, más elaborado y con sistemas más complejos, que incluía el uso del control remoto.

El desarrollo de juegos propios con consolas de distintas generaciones se da gracias a un proceso de aprendizaje y de investigación de las distintas tecnologías necesarias para elaborar juegos con desarrollos muchísimo más complejos. Después de fabricar algunas aplicaciones gratuitas para teléfonos móviles, su primer gran éxito alcanzó tres millones de descargas y obtuvo el primer puesto en el ranking de aplicaciones de paga más descargados de la iTunes App Store en México. Posteriormente, ganó el premio al Mejor Juego Móvil en los MTV Game Awards 2012 Latinoamérica. Debido al auge de los teléfonos móviles y las tablets, la empresa continúa en este campo con aplicaciones para Nokia, Apple, Windows, Windows Phone, Windows 8, Android; prácticamente en todos, excepto BlackBerry.

Si bien las TI han penetrado en la industria del entretenimiento y medios interactivos, la técnica es un requerimiento necesario pero no suficiente. El éxito depende, sobre todo, de la creatividad. El desarrollo de videojuegos, por ejemplo, involucra un equipo que cuente con programadores de software de primer nivel, pero también desarrollo de personajes, diseño y escenografía; en palabras del entrevistado, entre $80 \%$ y $90 \%$ por ciento del personal debe ser creativo. 
Además de los juegos, la empresa ha incursionado en el área de la animación (Kaxan Animation), con una cinta que se presentó en el Festival de Guadalajara, y en películas (Kaxan Studios).

La última área de la empresa, pero no por ello la menos importante, es Kaxan Campus, con la que se han establecido alianzas estratégicas con universidades. Con ello, buscan influir en la estructura curricular y tienen sedes en Chapala y en Vallarta, Jalisco, donde ofrecen cursos, algunos gratuitos. La idea detrás de esta labor es no sólo poder contratar los mejores elementos, sino también generar economías externas para una industria naciente que tiene gran potencial y que, en la medida que construya un nombre, será apreciada por los actores de la cadena de valor internacional, de la misma manera que la India es reconocida en el terreno del software.

El apoyo del Prosoft ha sido importante para la capacitación y equipamiento; asimismo, la empresa ha obtenido algunos apoyos a la innovación del CONACYT y está ahora ubicada en el centro de Guadalajara, en donde se planea dar cobijo a la industria creativa y digital. También han recibido apoyos estatales.

El financiamiento fue inicialmente propio; para el segundo año se hizo la primera ronda de inversión y está en proceso la segunda. Han participado con la aceleradora TechBA, cuyo apoyo ha sido importante para iniciar la construcción de las intrincadas redes que sustentan a la industria de ті y medios interactivos, sin las cuales, en palabras del entrevistado, no se puede tener éxito. Al respecto, agrega que Guadalajara tiene una ventaja importante porque hay numerosos vuelos a Los Ángeles por día, y a otras ciudades estadounidenses fundamentales en materia de eventos de las industrias creativas interactivas.

Para el entrevistado, se están creando capacidades y han surgido muchas empresas con aplicaciones para móviles y tablets o creadoras de películas. Pero el principal obstáculo para empresas como ésta sigue siendo la falta de capital de riesgo en nuestro país. En México, no sólo los bancos no prestan, sino que no hay capital de riesgo. Asegura que los programas que existen en el país no son competitivos en absoluto frente a los 200 fondos de riesgo que puede haber tan sólo en Silicon Valley, a los que pocas empresas mexicanas pueden acceder.

\section{CONCLUSIONES E IMPLICACIONES DE POLÍTICA}

El análisis de los casos reseñados permite mostrar varias semejanzas sugerentes de los condicionantes positivos para la acción de las empresas. Algunas comienzan con negocios dirigidos al mercado local, pasando al nacional y luego al in- 
ternacional, mientras que otras nacen con la percepción clara de una oportunidad de negocios de exportación, en particular en Estados Unidos.

Softtek es, tal vez, el caso más nítido al respecto y la empresa que tiene la más alta relación entre exportaciones y ventas. Las entrevistas muestran que la proximidad geográfica otorga a las empresas mexicanas de TI una ventaja debido a: 1) los costos reducidos de transporte y la posibilidad de viajar para encuentros frecuentes con los clientes y para hacer relaciones; 2) la menor diferencia horaria, que favorece un mayor contacto entre clientes y proveedores; 3) los beneficios para la inmigración otorgados en el marco del TLCAN (expedición de visas, por ejemplo, lo cual es útil para la relocalización de la subcontratación, y 4) el compromiso del gobierno mexicano con los acuerdos sobre ADPIC de la WIPO.

Sin duda, todos los casos son ejemplos de espíritu empresarial con visión de largo plazo y disposición a tomar riesgos, características que no siempre están presentes en nuestra economía. Puede decirse que en todas las empresas examinadas hay un esfuerzo consciente por avanzar en una senda de aprendizaje.

Respecto a la formación de las capacidades del equipo laboral, la mayor parte de las empresas manifestaron la necesidad de reclutar personas con un buen perfil de formación profesional. Sin embargo, esto no significa que esta formación sea suficiente y de ahí que sea necesario el entrenamiento fuera y dentro de la empresa. El trayecto hacia la certificación involucra capacitación en mejores prácticas. Para tres de las empresas, la certificación ha sido una herramienta fundamental para acceder a los mercados (Softtek, Delaware y Qualtop, la cual, a su vez, ahora se dedica a certificar empresas). Las otras consideraron que la certificación no es importante para sus mercados, pero sí el conocimiento de las herramientas y, en el caso de Kaxan, de un conjunto de conocimientos ligados al diseño y la creatividad, el cual sus empleados no tienen originalmente en grado suficiente. Varias de las empresas manifestaron su cercanía con universidades para impartir clases

Las entrevistas muestran que hay interacción considerable entre las empresas y sus clientes, lo cual quiere decir que no obstante la estandarización y las certificaciones, sigue habiendo necesidad de un intercambio de conocimiento tácito. Por otra parte, en cinco de las empresas, la adecuación del producto/servicio a las necesidades del cliente está presente. El caso de Kaxan es distinto porque vende directamente al consumidor final y sus proveedores serían los de consolas de desarrollo de las grandes firmas y los juegos o aplicaciones como segmentos independientes. 
Las empresas han aprovechado los apoyos del Prosoft, tres de ellas para las certificaciones y cuatro para equipamiento. Una empresa grande como Softtek pudo aprovechar el programa MexicofIRST para el desarrollo de capacidades y la iniciativa Pro México para promover exportaciones. Aun cuando la certificación IT LINK se encuentra en una etapa incipiente, tiene gran potencial, como se pudo observar en el caso de Qualtop. De hecho, los clientes de ésta tienen un fuerte apoyo del Prosoft en Guadalajara.

El papel que desempeñó TechBA también fue crucial en el caso de tres de las empresas de TI que estudiamos (Scio, Delaware y Kaxan). En algunos casos, su asistencia ha sido tan importante que se le ha considerado como una piedra angular para algunas de ellas. Da la impresión que este apoyo en asesoría es una precondición para desarrollar no sólo capacidades de negocio ordinarias sino capacidades de mercadotecnia internacional que tienden a estar ausentes en las empresas locales.

En el pasado, las críticas se centraron en la supuesta falta de coordinación entre programas e instituciones (Brown y Domínguez, 2010). En el caso de la industria del software, la coordinación no resulta evidente. Nuestros entrevistados elogiaron al Prosoft como un buen instrumento, sin embargo, agregaron que su desempeño depende en gran medida de los gobiernos locales. Esto puede observarse en los diversos niveles de éxito que atestiguan distintas zonas del país, pues mientras el desempeño de este programa es altamente encomiable en Jalisco, y particularmente en Guadalajara, no resulta igual en otros estados, como puede observarse en los casos de Qualtop y Scio. Esto podría sugerir fuertemente que el software no es una prioridad en todo México. Por ejemplo, la Ciudad de México, en donde es reconocido que hay talento y capacidades entre los egresados de las universidades y, además, un cúmulo de empresas intensivas en información, no se ha logrado configurar un conglomerado que potencie las economías de aglomeración y, por tanto, las empresas funcionan en forma relativamente aislada.

Los entrevistados de Softtek, Scio y Delaware señalaron, en su momento, que no habían logrado acceder a los apoyos del CONACYT para la innovación, los cuales dan la impresión de estar dirigidos a la creación de nuevos productos y que carecen de una comprensión adecuada del papel que desempeña el software y su potencial innovador. Esto sugiere que se requiere dar particular atención a esta industria, ya que podría no existir suficiente claridad entre los evaluadores o dictaminadores con respecto a qué quiere decir innovación en términos de software. De hecho, es posible que se requiera ampliar el diseño de programas espe- 
cíficos (dirigidos a ciertas características de esta cadena de valor) para que vaya más allá del fortalecimiento de la capacitación y la certificación. Como ya lo hemos señalado, otros países que compiten con México ya cuentan con estos programas específicos para la industria, como India y Corea del Sur.

Desde una perspectiva de política pública, también se encontró que reducir la burocracia gubernamental resulta fundamental. La cantidad de papeleo para acceder a los recursos del Prosoft es extremada y engorrosa, particularmente para las pequeñas empresas, que son las que más necesidad tienen de certificaciones ad hoc como la de MoProsoft en el caso del software. Esto tiene que mejorar.

Con la excepción de Softek, los entrevistados de las demás manifestaron que un gran obstáculo es la falta de crédito. En la opinión de los autores de este artículo, hay un desequilibrio notorio entre los esfuerzos para asegurar inversión por parte de empresas extranjeras y la certificación frente a iniciativas de financiamiento de largo plazo. Esto último se ha abordado de modo inapropiado y, por tanto, hay que considerarlo como una falla. La evidente deficiencia del sistema crediticio mexicano es excesiva: no obstante el sistema de garantías nacionales establecido en 2003, 97\% de los fondos para crédito están destinados a capital de trabajo. En otras palabras, el crédito de largo plazo es prácticamente inexistente (De Maria, Domínguez y Brown, 2010).

En el caso del software, el acceso a fondos es incluso más difícil. Debido a que las empresas de esa industria sólo pueden proporcionar garantías intangibles (capital humano y conocimiento), los inversionistas tradicionales y los bancos son muy proclives a rechazar las solicitudes de financiamiento. Si bien este problema podría ser endémico de las empresas de software en otros países, como Taiwán, la ausencia de capital de riesgo y de financiamiento de largo plazo en la banca de fomento mexicana constituye una desventaja competitiva en comparación con, por ejemplo, las empresas brasileñas, coreanas o españolas, por mencionar algunas que cuentan con atractivos programas para fomentar el emprendimiento. Deben establecerse políticas y mecanismos creativos para inducir a los bancos a que canalicen una parte significativa de sus recursos al financiamiento de equipo y desarrollo de tecnologías de hardware y software. Asimismo, es importante cambiar la política de la banca de fomento mexicana (Nafin), que a la fecha sigue dando preferencia el otorgamiento de crédito vía factoraje (para $60 \%$ de su portafolio). El cambio implicaría incorporar programas para proyectos de financiamiento del tipo que requieren las industrias de ID y software. Conscientes de que hay esfuerzos por atender algunos temas, los au- 
tores asumen que la promoción de empresas - extranjeras o locales- en estas áreas con los programas existentes en la Secretaría de Economía y CONACYT, incluso el financiamiento para certificación, es insuficiente. Sin crédito ni capital de riesgo competitivos, la inserción de las empresas mexicanas a las CVG podría quedar estancada en la trampa de los países de ingreso medio, como señala un interesante documento de varias instituciones internacionales en el cual se insiste en la necesidad de definir una estrategia de desarrollo con medidas selectivas centradas en la disección de las actividades de la cadena en finos segmentos (OECD, WTO y UNCTAD, 2013; Kowalski et al., 2015).

En opinión de los autores, las empresas de software mexicanas parecen no destacar en la competencia global, a pesar de que se hace evidente que las condiciones para ingresar están dadas y que dichas empresas están aceptando el reto. Hasta ahora, la mayor parte de las ellas participan como proveedores cautivos o en los niveles inferiores de valor agregado; sin embargo, los casos expuestos muestran que hay espacio para ascender en la cadena de valor. Para que esto se logre, dos condiciones son necesarias:

1) Los emprendedores deben evolucionar. Para avanzar en la producción de servicios con alto valor agregado con éxito, deben seguir invirtiendo en el desarrollo de capacidades y certificaciones. Asimismo, deben plantearse metas ambiciosas para conseguir clientes internacionales y encontrar nuevos nichos de mercado. La prevalencia de empresas muy pequeñas en la industria del software plantea la pregunta de si la idiosincrasia del emprendedor mexicano se ha convertido en un obstáculo infranqueable para alcanzar alianzas estratégicas y fusiones entre pequeñas empresas con el fin de crecer. Parece que entre los emprendedores hay cierta conciencia sobre la necesidad de unir fuerzas para competir en la arena internacional, como podría ser en el conglomerado de Monterrey, el IJALTI y alguna otra propuesta para formar un consorcio de empresas. Pero este tipo de iniciativas están muy lejos de ser la regla.

2) El gobierno debe diseñar una política más agresiva con una visión de largo plazo. Hay rasgos generales de política económica que han sido importantes para el crecimiento de esta CVG en el corto plazo. Un ejemplo sería el lanzamiento de un programa educativo de envergadura para mejorar la enseñanza de las matemáticas y el inglés, lo cual potenciaría las capacidades de los futuros empleados en diversas industrias, entre otras la de las TIC. Por otra parte, las lecciones que se pueden extraer de 
estos estudios de caso y la experiencia de otros países indican la necesidad de contar con programas específicos que respondan a las necesidades concretas de la industria de TI. Si se quiere ingresar en una CVG de tecnología de punta, las dependencias del gobierno tienen que superar los desafíos del desarrollo, que van más allá de tender puentes tradicionales entre las fallas del mercado. Estos desafíos requerirán resolver de una vez por todas el problema de la escasez de financiamiento y capital de riesgo para la industria y utilizar el poder de compra del gobierno, tal como lo hacen en la India o China, y fomentar proyectos de alto impacto que proporcionen infraestructura física, mejoren la logística y las condiciones de comunicación y, lo más importante, fomenten la innovación y las capacidades de aprendizaje entre los pioneros de estas nuevas cadenas, así como las alianzas estratégicas entre ellos. En otras palabras, gobierno y empresarios deben trabajar con una meta común.

\section{REFERENCIAS BIBLIOGRÁFICAS}

Arora, Ashish, y Gambardella, Alfonso (2004), "The Globalization of the Software Industry: Perspectives and Opportunities for Developed and Developing Countrie", NBER Working Paper 10538.

Brown, Flor, y Domínguez, Lilia (2010), "Políticas e instituciones de apoyo a la pequeña y mediana empresa en México", en Ferraro y Giovanni Stumpo Carlo (comps.), Políticas de apoyo a las pymes en América Latina: Entre avances innovadores y desafios institucionales, Santiago de Chile, CEPAL, pp. 299-341.

Delaware Software, www.delaware-software.com

De Maria, Mauricio; Domínguez, Lilia, y Brown, Flor (2010), "Mexican Industry at a Crossroads: Some Policy Considerations", Latin American Policy, 1 (2), pp. 284-306.

Dossani, Rafiq, y Kenney, Martin (2006a), “The Relocation of Service Providers to Developing Nations: The Case of India”, en A. Newman y J. Zysman, How Revolutionary Was the Digital Revolution, Stanford, Stanford University Press, pp.193-216.

_ (2006b), "Software Engineering: Globalization and its Implications", ponencia presentada al Workshop on the Offshoring of Engineering: Facts, Myths, Unknowns and Implications, 24 y 25 de octubre, Washington, DC.

Fernandez-Stark, Karina; Bamber, Penny, y Gereffi, Gary (2011), Skills for Upgrading: 
Workforce Development and Global Value Chains in Developing Countries, s.1., Duke University/Center on Globalization, Governance \& Competitiveness.

García, Pablo (2011), Base de conocimiento sobre el Prosoft 2.0, s.1., Consultores C230.

Gereffi, Gary; Humphrey, John, y Sturgeon, Timothy (2005), “The governance of global value chains", Review of International Political Economy, 12 (1), pp.78-104.

Greenfield, Jack, y Short, Keith (2003), Software Factories, Assembling Applications with Patterns, Models, Frameworks, and Tools, s.1., OoPSLA.

Heeks, Richard; y Nicholson, Brian (2002), "Software Export Success Factors and Strategies in Developing and Transitional Economies". Consultado durante junio de 2011, en: http://www.man.ac.uk/idpm/idpm_dp.htm\#devinf_wp.

Hualde, Alfredo, y Mochi, Prudencio (2008), "México: ¿una apuesta estratégica por la industria del software?", Comercio Exterior, 58 (5), pp. 335-349.

Ju, Dehua (2001), “China's Budding Software Industry”, IEEE Software, 18 (3), pp.92-95. Kaplan, G. (1998), “Israel: A High-Tech Haven”, IEEE Spectrum 35 (5), pp. 22-32.

Kowalski, Przemyslaw; López González, Javier; Ragoussis, Alexandro, y Ugarte, Cristian (2015), "Participation of Developing Countries in Global Value Chains: Implications for Trade and Trade-Related Policies", OECD Trade Policy Papers 179.

Lall, Sanjaya (2000), “The technological structure and performance of developing country manufactured exports", Oxford Development Studies, 28 (3), pp.337-369.

Leamer, Edward E., y Storper, Michael (2001), "The Economic Geography of the Internet Age", NBER Working Paper 8450.

López, Andrés; Ramos, Daniela, y Torre, Iván (2010), “América Latina en las cadenas globales de valor en servicios: ¿Se puede ir más allá de generar divisas y empleos?”, Innovación y Conocimiento, segunda época 5 (2), pp.227-251.

Makarov, Valentín (2003), “The Russian Software Industry”, presentación como presidente de Russoft.

Mejía, Marcelo; Ania, Ignacio, y Gamboa, Rafael (2006), "Diagnóstico de la industria de servicios de software en México", AMCIS 2006, proceedings paper 497 [en línea]. Consultado durante septiembre de 2011, en: http://aisel.aisnet.org/amcis2006/497.

Mochi, Prudencio y Alfredo Hualde (2009), México: producción interna e integración mundial, desafios y oportunidades de la industria de software en América Latina, Santiago de Chile, CEPAL.

Moitra, Deependra (2001), "India’s Software Industry", IEEE Software 18 (1), pp.77-80. Mullan, Jessica; Kenney, Martín, y Dossani, Rafiq (2008), "Mexico and the Globaliza- 
tion of Services: Outflanked Once Again?", Economía Mexicana, nueva época 17 (2), pp.171-202.

OECD, UNCTAD y wTo (2013), "Implications of Global Value Chains for Trade, Investment, Development and Jobs", documento de trabajo para la cumbre del G-20.

Qualtrop. www.qualtop.com/

Salmenkaita, Jukka-Pekka, y Salo, Ahti (2002), "Rationales for Government Intervention in the Commercialization of New Technologies", Technology Analysis \& Strategic Management, 14 (2), pp.183-200.

Sánchez, Ron, y Collins, Robert (2001), "Competing and Learning in Modular Markets", Long Range Planning, 34 (6), pp. 645-667.

Saxenian, Anna Lee, y Hsu, Jin-Yuh (2001), "The Silicon Valley-Hsinchu Connection: Technical Communities and Industrial Upgrading”, Industrial and Corporate Change, 10 (4), pp. 893-920.

Schatan, Claudia, y Enríquez, Leobardo (2012) "La producción de bienes y servicios de tecnologías de la información y la comunicación y las políticas industriales en México", mimeo.

Schilling, Melissa (2000), “Toward a General Modular Systems Theory and Its Application to Interfirm Product Modularity", Academy of Management Review, 25 (2), pp. 312-334.

Secretaría de Economía (2012), "Prosoft 2.0, Programa de Desarrollo del Sector de Servicios de Tecnologías de Información", México, Secretaría de Economía [pdf en respaldo físico].

Software Top 100 (2011), "The World's Largest Software Companies". Consultado el durante mayo de 2013, en: www.softwaretop100.org/global-software-top100-edition-201.

Stephenson, Sherry (2012), Emergence of Services, Global Value Chains (GVC) and Resulting Implications for Latin America, s.l., Department of Economic Development/Trade and Tourism Executive Secretariat for Integral Development.

Terekhov, Andrey A. (2001), “The Russian Software Industry”, IEEE Software, 18 (6), pp. 98-101.

Wibe, Mona Dommas, y Narula, Rajneesh (2004), "Interactive learning and non-globalisation: knowledge creation by Norwegian software firms", International Journal of Entrepreneurship and Innovation Management, 2 (2-3), pp. 224-245.

Zermeño, Ricardo (2011), "Estructura y dimensiones de la industria TIC", Política Digital, 10 (63), pp. 46-51. 